\title{
Agarose floor technique: a simple scaffold-free method for 3D cell culture and multicellular tumor spheroids formation.
}

César Rivera, B.Dent.Sc., D.D.S., M.Sc.

From the Department of Basic Biomedical Sciences, University of Talca (UTALCA), Talca, Maule Region, Chile; the Department of Oral Diagnosis, School of Dentistry (FOP), University of Campinas (UNICAMP), Piracicaba, São Paulo, Brazil; Mass Spectrometry Laboratory, Brazilian Biosciences National Laboratory (LNBio), National Center for Research in Energy and Materials (CNPEM), Campinas, São Paulo, Brazil.

Supported by: CR is beneficiary of Chile's National Commission for Scientifc and Technological Research (CONICYT) Becas-Chile scholarship for Ph.D. students No. 8540/2014.

Address correspondence to: Jaime Rodríguez Carvajal Building, University of Talca (UTALCA), Lircay Av. S/N, Talca, Maule Region, 3460000, Chile (contacto@ cesarrivera.cl).

Keywords: agarose, tumor spheroid models, three-dimensional tumor spheroids. 


\section{To the editors:}

3D cell cultures can close the gap between in vitro experiments used for discovery and in vivo designs used for efficacy and safety assessment before proceeding to clinical studies ${ }^{1}$. 3D multicellular tumor spheroids reflect better the tumor environment in terms of phenotypic heterogeneity, nutrient and oxygen gradients, intervascular domains, and micrometastases ${ }^{2}$.

This letter presents the agarose floor technique to obtain single-cell suspensions from oral cancer cells in nonadherent conditions. SCC-9 cells (American Type Culture Collection, Manassas, VA, USA) were cultured in culture plastic wares with nonadhesive surface. $150 \mathrm{~mm}$ dish are made of nonadhesive for cells by coating with $3.2 \%$ sterile agarose (Analytical Grade, Promega,

Madison, WI, USA) thin films (5-8 mL/dish). This agarose floor is allowed to dry before addition of medium (DMEM/Ham's F12, supplemented with $10 \%$ fetal bovine serum, antibiotics and 0.4 $\mu \mathrm{g} / \mathrm{mL}$ hydrocortisone) containing $6 \times 10^{5}$ live cells/dish. The cells were maintained at $37^{\circ} \mathrm{C}$ in a $5 \%$ $\mathrm{CO}_{2}$ atmosphere and the culture medium was changed every 2 days until the sphere formation (8 days). With the passing of the days multicellular tumor spheroids are obtained. The spheres were examined with microscope and digital camera (Figure 1).

Multicellular tumor spheroids are easily generated by the liquid overlay technique that prevents matrix deposition. Tumor cells are placed on tissue culture plastic covered with a thin layer of inert substrate such as agarose ${ }^{3}$. Agarose is frequently used in molecular biology for the separation of large molecules, especially DNA, by electrophoresis. The pore size of a 3\% agarose gel has been estimated in $\sim 290 \mathrm{~nm}^{4}$. The low porosity, along with being an inert material, make the agarose a good candidate to obtain a non-adherent surface to epithelial cells. 


\section{REFERENCES}

[1] Zanoni M, Piccinini F, Arienti C, Zamagni A, Santi S, Polico R, Bevilacqua A, Tesei A: 3D tumor spheroid models for in vitro therapeutic screening: a systematic approach to enhance the biological relevance of data obtained. Sci Rep. 2016;6:19103.

[2] Wang J, Zhang X, Li X, Zhang Y, Hou T, Wei L, Qu L, Shi L, Liu Y, Zou L, Liang X: Antigastric cancer activity in three-dimensional tumor spheroids of bufadienolides. Sci Rep.

2016;6:24772.

[3] Weiswald LB, Bellet D, Dangles-Marie V: Spherical cancer models in tumor biology. Neoplasia. 2015;17(1):1-15.

[4] Pernodet N, Maaloum M, Tinland B: Pore size of agarose gels by atomic force microscopy. Electrophoresis. 1997;18(1):55-8. 


\section{SCC9 cells}
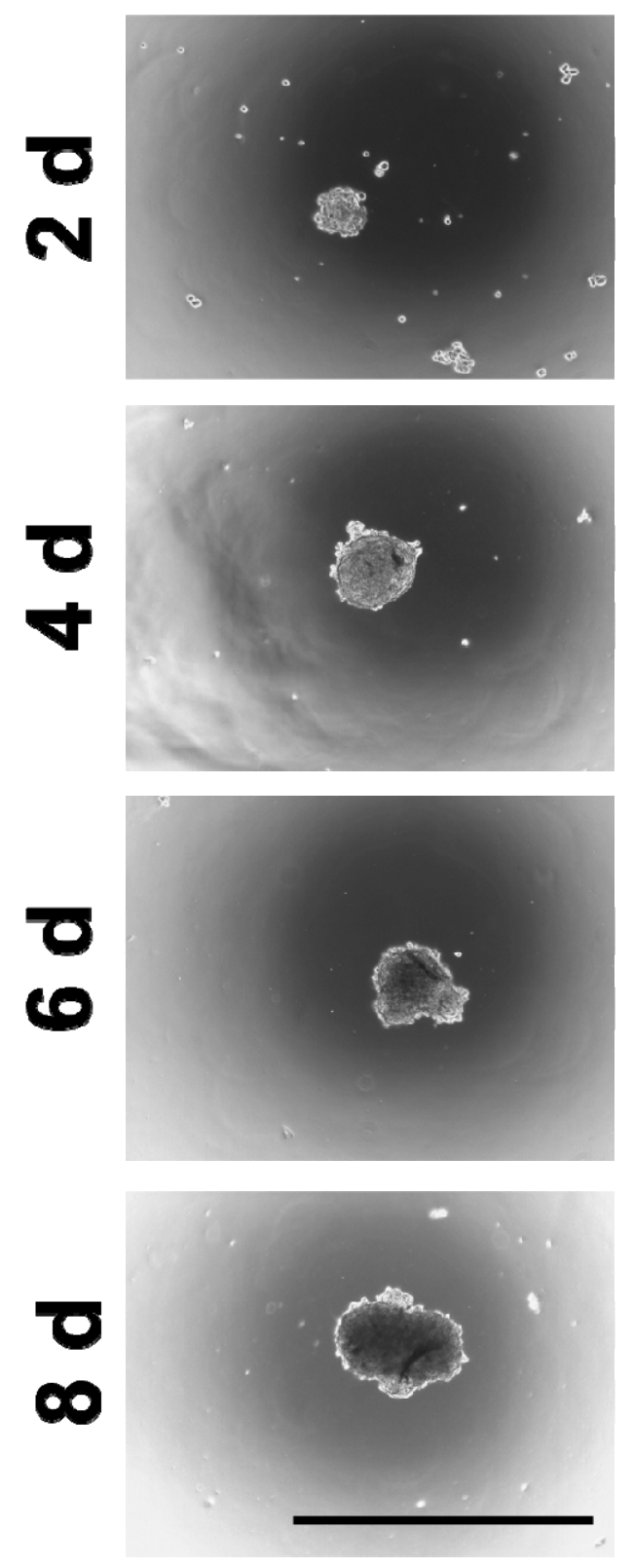

Figure 1. Three-dimensional multicellular tumor spheroids formation. Oral cancer cells (SCC9) grown in non-adhesive liquid-based culture tend to form cell clusters (panels from day 2 to 8 ). With tumor spheroids, the cells appear fused together as "floating popcorns in the night sky". Scale bar, $1 \mathrm{~mm}$. 